Proceedings of the Online Conference "Applications of Physics in Mechanical and Material Engineering"

\title{
Probabilistic Model for Passage Time of Chain-Like Structure through Thick Membrane
}

\author{
Z. Domański* AND A.Z. Grzybowski
}

Częstochowa University of Technology, 42-201 Częstochowa, Poland

Doi: 10.12693/APhysPolA.139.513

*e-mail: zbigniew.domanski@pcz.pl

\begin{abstract}
Transfer of a polymer across a membrane is the subject of extensive theoretical and experimental works. One of the most fruitful approaches to such studies relies on the usage of Monte Carlo simulations. In those methods, the polymers are viewed as an abstract chain-like structure, and among the principal goals of the simulation-analysis is to relate their passage time through a cell boundary with various characteristics describing both the structures and their environment. In this paper, we develop probabilistic models for a passage time of the chain-like-structures through a thick membrane. We examine the impact of the boundary thickness on the passage time through a flat membrane as well as its dependence on the polymer length. All our experiments are carried out with the help of our own-developed sequential algorithm that incorporates the tension-propagation mechanism.
\end{abstract}

topics: Monte Carlo simulations, polymer translocation, sequential algorithm, thick membrane

\section{Introduction}

An abstract chain-like structure (CLS) plays an important role in modeling systems of different nature of ingredients with different types of interactions among them. The examples are electrically charged assemblies of nanoparticles, chainlike bodies of magnetic moments with different ordering, chain structures in poly-disperse ferrofluids, or the primary structure of proteins. Perhaps the best known bodies of such kind are the macromolecules [1], and especially the polymers. If a rigorous description of chemical details of the polymer is not necessary to capture the essential features of the biomolecule, a widely employed approach relies on a coarse-grained model of a polymer chain. Such a chain comprises a number of substituents called beads being the images of the complicated chemical monomers lying around the backbone of the real macromolecule.

Numerical computational schemes used in the field of polymer dynamics generally fall into two classes: (i) a molecular dynamics simulation when an appropriate second-order differential equation of motion is written down, such as the Langevin equation $[2,3]$ and then this equation is integrated in time by some methods [4] or (ii) a Monte Carlo experiments with implemented rules for the stochastic generation of a sequence of configurations that are used to trace the system trajectory in the configuration space [5]. Within these two classes of methods, the system is studied with regard to some constraints imposed either by a computational scheme alone or those coming directly from the field of study $[6,7]$.

Apart from the well-established requirements such as ergodicity or self-avoidance, a modern algorithm dedicated to studies within polymer science should also take into account the presence of the tension-propagation mechanism reflecting the response of a polymer to local drag forces and mutual segments interactions $[8,9]$. Thus, it is desired that any algorithm designed to study the dynamics of a polymer should contain an inherent computational pathway ensuring such mechanism.

The development of our own sequential algorithm was motivated by the conspicuous absence, within known algorithms, of a procedure which with high acceptance probability yields globally deformed conformations, yet fulfilling the self-avoidance and the ergodicity requirements and additionally supporting the tension mechanism. This algorithm works with a standard self-avoiding phantom polymer model on a 2D square lattice. The features of the sequential algorithms were studied and described in more detail in [10-12].

In our current studies, this algorithm is used to examine the CLS passage through a thick membrane. The majority of studies of this phenomenon concerned the case where the thickness of the membrane was very small or negligible, but there is still a growing interest in such cases, where the 
membrane is thick in comparison with the CLS length [13-18]. In this paper, we focus on the relationship between the thickness of the membrane and the distribution of the passage time.

The paper is organized as follows. In the next section, our Monte Carlo experiments are described in more detail. Section 3 proposes the Moyal probability distribution (p.d.) for modeling the passage times through a long pore in a thick membrane within the considered framework. Next, in Sect. 4, we present exemplary models that show the relationship between the parameters of the p.d. of the passage time and the membrane thickness. The article is concluded with some final remarks.

\section{Description of Monte Carlo experiments}

In our simulations of the self-avoiding polymer, we analyze a fluctuating-bond model in 2D. An abstract $2 \mathrm{D}$ position of the CLS is a finite sequence of $2 \mathrm{D}$ points (with integer coordinates) such that the distance between any pair of its successive elements is bounded by given limits. The elements of such a CLS-sequence are called segments, and their number is the length $(N)$ of the CLS. Within our algorithm, any translocation from one position of the CLS to another is achieved via a sequence of random allowable translocations of its segments that are called steps [10]. To simulate the imposed driving force, the sequential algorithm enables us to tune a bias on probable directions of random translocations of the CLS segments. In the considered case, such a small bias was imposed to drag the polymer segments along a pore between cis and trans sides of the membrane (see Fig. 1).

One can find in the literature various assumptions concerning the initial position of the polymer. In our experiments, we organize the CLS initial position in such a way that only the head of the CLS is placed in the opening of the membrane.

Within this scenario, we have collected large data sets containing records of translocation time $(\tau)$ for varying $N$ and membrane thicknesses $(L)$,

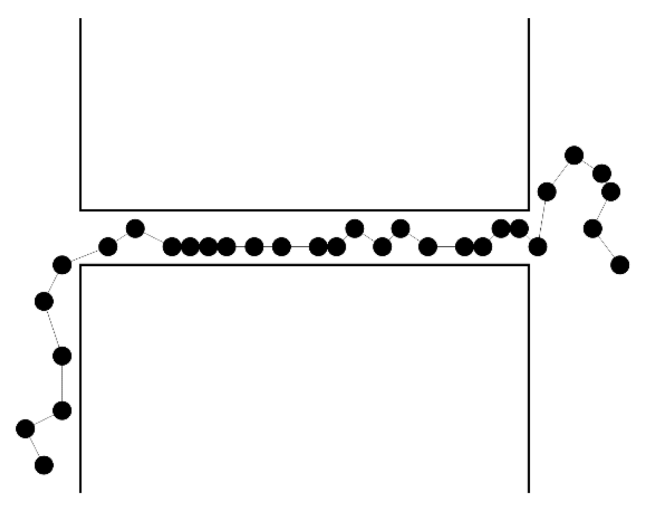

Fig. 1. A sketch of a polymer translocating through a long pore in thick membrane. with $100 \leq N \leq 200$ and $5 \leq L \leq 100$. During the simulations, we assumed that distances between successive segments are bounded by $\Delta_{\min }=2$ and $\Delta_{\max }=4$. In our case of the thick membrane, the pore in it can be thought of as a long tube whose diameter $\delta=2,3,4$. The geometrical characteristics $L, \Delta_{\min }, \Delta_{\max }$ and $\delta$ are expressed in unit being the underlying lattice constant. The translocation time is measured in steps, i.e., $\tau$ is the number of all single translocations made by all segments during the passage process.

\section{Probabilistic model for passage time}

When we attempted to model the randomness of $\tau$ with the help of a number of reasonable candidate-distributions, it turned out that the data received in our experiments very well followed one specific family of probability distributions, namely the family of Moyal distributions. It is worth mentioning that the family of Moyal distributions was already reported in [10], in the context of data related to translocations through a thin membrane. We can find there the descriptions of many - reported in the literature - experimental and simulation data concerning the passage times that can be very accurately modeled with the help of the Moyal distributions. Now, it results from our current experiments that the Moyal distribution is a very good probabilistic model for the passage time in the case of the thick membrane. Thus, the distribution of $\tau$ can be well approximated by a density function:

$$
p(\tau)=\frac{1}{s \sqrt{2 \pi}} \exp \left(-\frac{\mathrm{e}^{-(\tau-\mu) / s}}{2}-\frac{\tau-\mu}{2 s}\right),
$$

where $\mu$ and $s$ are the location and scale parameters.

\section{Impact of membrane thickness} on passage time distribution parameters

In this section, we present the regression models relating the membrane thickness $L$ and the Moyal p.d. parameters. These particular models are developed based on the data obtained for the CLS of the length $N=100$.

The differences between the theoretical and empirical (observed) distributions, which can be noticed in Fig. 2, are natural, and they are small in the statistical sense. All tests that we adopt here, such as the Anderson-Darling, the Cramér-von Mises, or the Kolmogorov-Smirnov, indicate a very high observed significance level. Thus, they strongly support our research thesis about the probability distribution of $\tau$.

Figure 3 shows the dependence of the mean passage time $\langle\tau\rangle$ on the membrane thickness $L$. The curves were obtained for three values of the pore-diameter $\delta$. As we see, within the observed simulation-parameters' ranges, the relationship between the expected value of $\tau$ and the membrane 


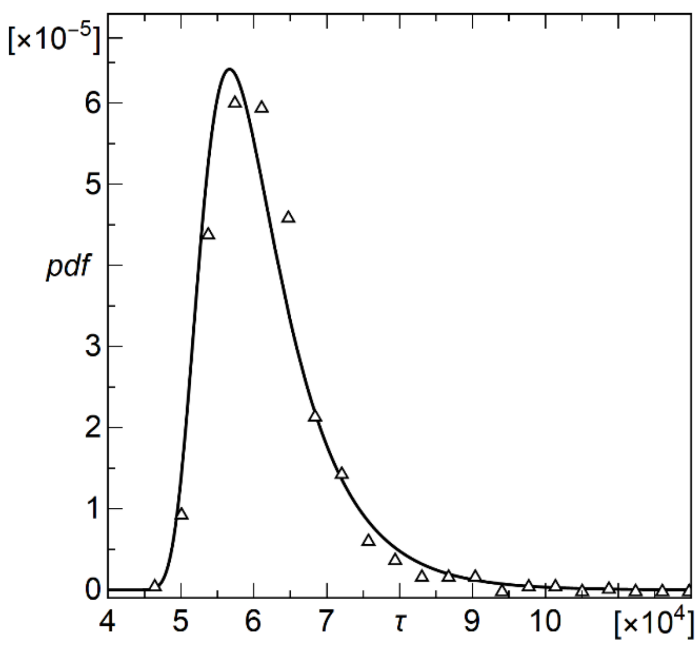

Fig. 2. The calculated distribution of $\tau$ for $L=100$ and $\delta=3$. The sample size is equal to $10^{3}$. The solid line is drawn according to (1) with parameters estimated from the simulations.

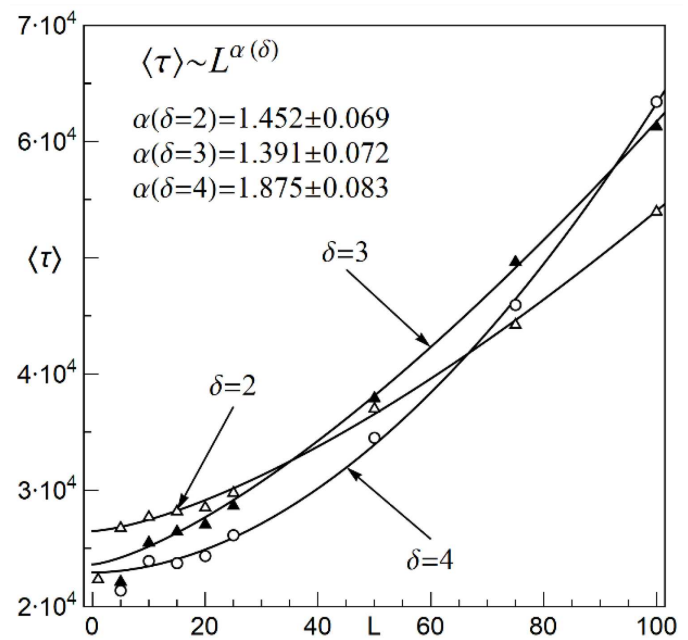

Fig. 3. Mean passage time $\langle\tau\rangle$ vs. the pore length $L$ for different pore diameters $\delta$ and polymers with $N=100$ segments. Lines represent the power law $\langle\tau\rangle \sim L^{\alpha}$ which is the best fit for the data. Results were obtained from 500 samples for each data point. The errors of $\alpha(\delta)$ indicate the limits of $95 \%$ confidence intervals.

thickness $L$ can be very well approximated by the power law relation $\langle\tau\rangle \sim L^{\alpha(\delta)}$, with the exponent $\alpha$ which depends on the pore diameter $\delta$ as well as on the polymer length $N$.

\section{Final remarks}

Our experiments clearly show that the mean $\langle\tau\rangle$ is in a power-law relation with the membrane thickness $L$ regardless of the pore diameter $\delta$. In view of the literature, that conclusion seems to be rather natural and - as such - the expected one.
However, what is perhaps less expected is that the slope of the model curves increases the faster, the larger the pore diameter. It implicates that for small thicknesses $L$, the average passage time $\langle\tau\rangle$ is the less, the bigger is the diameter $\delta$ [19]. But when the $L$ gets big enough, the relationship becomes the opposite. We can observe this phenomenon in Fig. 3. When we compare the mean passage time $\langle\tau\rangle$ for $\delta=2$ and $\delta=4$, we see that such a limit-thickness is about 70, i.e., if $L$ is less than 70 , then the mean passage time is greater for $\delta=2$. Yet if $L$ is greater than 70 , then the mean passage time is greater for $\delta=4$.

This phenomenon can be explained by the fact that for wider openings there is a chance for the CLS to form clusters of segments that can stick in the pore. Such clusters slow down the CLS transition, and the possibility of their creation is the greater, the thicker is the membrane and thus the longer is the pore. On the other hand, when the diameter of the pore is less than the minimum length of the bond, in our experiment $\delta<\Delta_{\min }=2$, clusters of segments cannot be created regardless of the membrane thickness. Obviously, such clusters cannot be observed for very wide openings either. Thus, one of the interesting future goals of research would be to determine $\delta$ 's value for which the model-slopeincrease is the fastest, and then to find the relationship between these values of $\delta$ and the length of the CLS as well as the membrane thickness.

\section{References}

[1] S. Kumar, M.S. Li, Phys. Rep. 486, 1 (2010).

[2] I. Huopaniemi, K. Luo, J. Chem. Phys. 126, 124901 (2006).

[3] M. Muthukumar, C.Y. Kong, PNAS 103, 5273 (2006).

[4] D.L. Ermak, H. Buckholz, J. Comput. Phys. 35, 169 (1980).

[5] H.-P. Hsu, W. Paul, Comput. Phys. Commun. 182, 2115 (2011).

[6] J.M. van Leeuwen, A. Drzwiński, Phys. Reports 475, 53 (2009).

[7] S. Żurek, M. Kośmider, A. Drzewiński, J.M. van Leeuwen, Eur. Phys. J. E 35, 47 (2012).

[8] T. Sakaue, Phys. Rev. E 76, 021803 (2007).

[9] J.L.A. Dubbeldam, V.G. Rostiashvili, A. Milchev, T.A. Vilgis, Phys. Rev. E 85, 041801 (2012).

[10] A.Z. Grzybowski, Z. Domański, arXiv:1312.4206v1, 2013.

[11] K. Bartłomiejczyk, J. Appl. Math. Comput. Mech. 17, 5 (2018). 
[12] Z. Domański, A.Z. Grzybowski, in: Proc. of 2015 IEEE 13th Int. Scientific Conf. on Informatics, Poprad (Slovakia), IEEE, 2015, p. 97.

[13] V.V. Palyulin, T. Ala-Nissila, R. Metzler, Soft Matter 10, 9016 (2014).

[14] K. Nagarajan, S.B. Chen, Macromol. Theory Simul. 29, 2000042 (2020).

[15] M.N. Hamidabad, R.H. Abdolvahab, Sci. Rep. 9, 17885 (2019).
[16] R.H. Abdolvahaba, M.N. Hamidabad, Eur. Phys. J. E 43, 76 (2020).

[17] S. Buyukdagli, J. Sarabadani, T. AlaNissila, Polymers 11, 118 (2019).

[18] Tingting Sun, Yunxin Gen, Hujun Xie, Zhouting Jiang, Zhiyong Yang, BioMed Res. Int. 2017, 5267185 (2017).

[19] Y.-D. He, H.-J. Qian, Z.-Y. Lu, Z.-S. Li, Polymer 48, 3601 (2007). 